\title{
Area-level socioeconomic disparity trends in nutritional status among 5-6-year-old children in Israel
}

\author{
Yiska Loewenberg Weisband 다, ${ }^{1}$ Vered Kaufman-Shriqui, ${ }^{2}$ Yael Wolff Sagy, \\ Michal Krieger, ${ }^{1}$ Wiessam Abu Ahmad, ${ }^{1}$ Orly Manor ${ }^{1}$
}

\begin{abstract}
- Additional material is published online only. To view please visit the journal online (http://dx.doi.org/10.1136/ archdischild-2019-318595)

'Braun School of Public Health and Community Medicine, Hebrew University of Jerusalem Faculty of Medicine, Jerusalem, Ein Karem, Israel

${ }^{2}$ Department of Nutritional Sciences, Ariel University, Ariel, Israel
\end{abstract}

\section{Correspondence to}

Dr Yiska Loewenberg Weisband, Braun School of Public Health and Community Medicine, Hebrew University of Jerusalem Faculty of Medicine, Jerusalem 91120, Israel

yiska.loewenberg@mail.huji.ac.il

Received 25 November 2019 Revised 17 February 2020 Accepted 12 April 2020 Published Online First 6 May 2020

\section{ABSTRACT}

Objective This study aimed to assess area-level socioeconomic position (SEP) disparities in nutritional status, to determine whether disparities differed by sex and to assess whether nutritional status and disparities changed over time.

Design We used repeated cross-sectional data from a national programme that evaluates the quality of healthcare in Israel to assess children's nutritional status. Setting The study included all Israeli residents aged 7 years during 2014-2018 ( $n=699255)$.

Methods SEP was measured based on the Central Bureau of Statistics' statistical areas, and grouped into categories, ranging from 1 (lowest) to 10 (highest). We used multivariable multinomial regression to assess the association between SEP and nutritional status and between year and nutritional status. We included interactions between year and SEP to assess whether disparities changed over time.

Results Children in SEP 1, comprised entirely of children from the Bedouin population from Southern Israel, had drastically higher odds of thinness compared with those in the highest SEP (Girls: OR 5.02, 99\% Cl 2.23 to 11.30 ; Boys: OR 2.03, $99 \% \mathrm{Cl} 1.19$ to 3.48 ). Odds of obesity were highest in lower-middle SEPs $\left(\mathrm{OR}_{\mathrm{SEP} 5 \text { VS } 10} 1.84,99 \% \mathrm{Cl} 1.34\right.$ to 2.54). Prevalence of overweight and obesity decreased between 2014 and 2018, normal weight increased and thinness did not change. SEP disparities in thinness decreased over time in boys but showed a reverse trend for girls. No substantial improvement was seen in SEP disparities for other weight categories.

Conclusions Our study demonstrates the need to consider initiatives to combat the considerable SEP disparities in both thinness and obesity.

\section{INTRODUCTION}

Childhood overweight and obesity (ie, high body mass index (BMI) for age and sex) are associated with adverse life-course health. ${ }^{12}$ Childhood thinness (ie, low BMI for age and sex) also imposes substantial consequences for child development including stunting, a weakened immune system and disability later in life. ${ }^{34}$

Early childhood is a critical period for detecting children at risk of overweight, obesity and undernutrition. School-aged children with obesity are 6.5 times more likely to become adults with obesity compared with children without obesity, ${ }^{5}$ while children with persistently low BMI through infancy,

\section{What is already known on this topic?}

- Individual-level socioeconomic position is inversely associated with childhood obesity in high-income countries.

- Mixed results have been found when assessing the association between individual level socioeconomic position and thinness.

- Mixed results have also been found when assessing the impact of area-level socioeconomic position on childhood bodymass index.

\section{What this study adds?}

- Overweight and obesity prevalence rates decreased over time, normal weight increased and no change was found for thinness prevalence.

- Major disparities in childhood thinness were found using area-level measures of socioeconomic position, with highest thinness rates found for children in the lowest socioeconomic position.

- Substantial disparities were observed in obesity rates, with highest obesity rates found for children in the lower-middle socioeconomic positions. These disparities did not improve over time.

who have a rapid BMI increase in later childhood, have a 30\% higher risk of developing type 2 diabetes mellitus in adulthood. ${ }^{6}$

Individual-level socioeconomic position (SEP) is inversely associated with obesity across highincome countries. ${ }^{7}$ Few studies in high-income countries have assessed the association between SEP and thinness, and these have found mixed results. ${ }^{89}$ Conflicting results have also been found regarding the association between area-level SEP and childhood BMI. ${ }^{10-12}$

Between-country comparisons of children's nutritional status is challenging due to the use of several definitions and reference cut-off points (Centers for Disease Control and Prevention, WHO and International Obesity Task Force growth references) and different terms (underweight, wasting, thinness, undernutrition). ${ }^{13}$ In this paper, we have used the 
terms thinness, overweight and obesity using the WHO reference population. $^{14}$

Our study aimed to assess the prevalence of thinness, normal weight, overweight and obesity among Israeli children, to assess area-based SEP disparities in nutritional status and to determine whether these disparities differed by sex. In addition, we aimed to assess trends in nutritional status over time and to examine whether SEP disparities differed over time.

\section{METHODS}

The Israel Quality Indicators in Community Healthcare (QICH) programme is a national programme that monitors and evaluates the quality of community-based healthcare provided through Israel's four health maintenance organisations (HMOs) using data originating from electronic medical records, for the entire Israeli population. ${ }^{15}$ Our study used QICH data to assess the nutritional status of Israeli children.

\section{Population}

The study population included all children aged 7 years in 20142018 (data included distinct cohorts of children aged 7 for every given year). In 2014-2015, the population was limited to three of the four HMOs, covering $92 \%$ of the children's population in that period. From 2016, data were available from all four HMOs, covering all children who were residents of Israel. The data do not include children with incomplete membership in an HMO during the study period ( $<2 \%$ of the population). ${ }^{15}$ Finally, we excluded children with a missing SEP from our analysis $(n=29719)$. A total of 699255 children were included in the study.

\section{Data}

For the current study, we used quality indicators from the QICH programme, regarding childhood nutritional status. All the indicators in the QICH programme are calculated by the HMOs, using individual-level information, and then anonymised and aggregated before they are shared with the QICH programme. Weight and height are routinely measured in children aged 5-6 in all Israeli HMOs; measurements are taken and recorded by trained staff using a published protocol of the Israeli Ministry of Health. ${ }^{16}$ The HMOs calculated the proportion of children aged 7 years with documentation of at least one measurement of height and weight during the past 2 years (when the children were ages 5-6). For children with more than one height and weight measurement available during the relevant time period, the most recent height and weight measurements were used to determine their BMI(weight in kilograms divided by the square of the child's height in metres). Nutritional status was determined by the HMO using WHO growth standards to classify sex-age-specific BMI z-scores for each child, using the child's sex and exact age. BMI z-scores and cut-off values were calculated using WHO guidelines. ${ }^{17}$ Data were calculated individually and transferred to QICH aggregated by age, sex and BMI z-score category. Four BMI categories were used: thinness (BMI z-score $<-2$ SD), normal weight (BMI z-score $\geq 2$ SD and $<+1 S D$ ), overweight (BMI z-score $\geq+1 \mathrm{SD}$ and $<+2 \mathrm{SD}$ ), obesity (BMI $\mathrm{z}$-score $\geq+2 \mathrm{SD})$.

\section{Socioeconomic position (SEP)}

SEP was based on the small statistical areas (SSA) used in the 2008 Israeli census. SSAs contain 3000-4000 people and are created in a way that maintains homogeneity in terms of the sociodemographic makeup of the population. ${ }^{18}$ The Central Bureau of
Statistics (CBS) used information regarding demography, education, employment, housing conditions and household income to define the SSAs, and these were grouped into 20 categories. ${ }^{18}$ As the latest data available from the CBS was from 2008, we used data updated by the POINTS Location Intelligence Company to improve the accuracy of the SEP measure, using current sociodemographic, commercial and housing data. ${ }^{19}$ These data were grouped into 10 categories, ranging from 1 (lowest) to 10 (highest). SEPs 1-2 (the lowest SEPs) consist predominantly of Arab neighbourhoods, with SEP 1 consisting exclusively of the Bedouin population of Southern Israel. SEPs 6-10 are predominantly Jewish, while SEPs 3-5 consist of a mix of Jewish and Arab neighbourhoods (Personal communication with POINTS Location Intelligence Company).

\section{Statistical analysis}

We report findings of nutritional status using proportions, by sex and SEP for the most recent year (2018) and nutritional status prevalence for every year available. As our outcome of interest was categorical, we used multivariable multinomial regression models, adjusted for sex, to assess the association between SEP and nutritional status for every year from 2014 to 2018 . Normal weight was used as the reference category.

We first assessed whether the trend of the association between SEP and nutritional status was monotonic. If monotonic, we compared the most extreme SEP groups. ${ }^{20}$ We found a monotonic trend for thinness and overweight, and we therefore compared the lowest and highest SEPs (1 vs 10) to assess SEP disparities in these categories. In obesity, instead of a monotonic trend, we found a reverse U-shaped association and therefore included a quadratic term for SEP to the model. Goodness of fit of the quadratic model was assessed using a likelihood ratio test. To assess SEP disparities in this category, we compared the lower-middle SEP to the highest (5 vs 10), as SEP 5 consistently had the highest prevalence of obesity.

In order to assess whether the association between SEP and nutritional status differed by sex, we included an interaction between sex and SEP. As we found a significant interaction between sex and SEP, we presented results stratified by sex.

We used a logistic regression model, adjusted for SEP and sex, to assess whether the prevalence of nutritional status changed over time, allowing us to assess the trend for thinness, normal weight, overweight and obesity separately (multinomial model showed similar trends). We used a multivariable multinomial regression model, including an interaction between year (as a continuous variable) and SEP, to assess whether SEP disparities differed over time.

Due to the large sample size, we based statistical significance on a p value of 0.01 and reported 99\% CIs. Analyses were performed using Stata V.14.0 (Stata, College Station, Texas, USA).

\section{Ethical standards}

The manuscript does not contain clinical studies or patient data.

\section{RESULTS}

Documentation of height and weight among 5-6 years old preschoolers was high overall, and ranged from $70.4 \%$ to $92.4 \%$ in boys, and from $71.2 \%$ to $91.2 \%$ in girls in 2018 (data not shown). The documentation rate in the lowest SEP was $91.8 \%$, while the documentation rate for children in the highest SEP was $70.8 \%$. Overall, documentation was available for $78.6 \%$ of the 148540 children in 2018, yielding 116774 children. A total of 546848 children were included for 2014-2018. SEP was not 
Table 1 Nutritional status of Israeli children aged 5-6 in 2018, by socioeconomic position and sex ( $n=116774$ )

\begin{tabular}{|c|c|c|c|c|c|c|c|c|c|c|}
\hline & \multicolumn{2}{|l|}{ Total } & \multicolumn{2}{|c|}{ Thinness } & \multicolumn{2}{|c|}{ Normal weight } & \multicolumn{2}{|c|}{ Overweight } & \multicolumn{2}{|c|}{ Obesity } \\
\hline & $\mathrm{N}$ & $\%$ & $\mathrm{~N}$ & Rate (\%) & $\mathrm{N}$ & Rate (\%) & $\mathrm{N}$ & Rate (\%) & $\mathrm{N}$ & Rate (\%) \\
\hline Total & 116774 & & 4986 & 4.3 & 90451 & 77.4 & 13206 & 11.3 & 8131 & 7.0 \\
\hline \multicolumn{11}{|c|}{ SEP } \\
\hline 1 & 4465 & 3.8 & 353 & 7.9 & 3540 & 79.2 & 356 & 8.0 & 216 & 4.8 \\
\hline 2 & 9803 & 8.4 & 589 & 6.0 & 7728 & 78.8 & 940 & 9.6 & 546 & 5.6 \\
\hline 3 & 17776 & 15.2 & 1025 & 5.8 & 13614 & 76.6 & 1913 & 10.8 & 1224 & 6.9 \\
\hline 4 & 15302 & 13.1 & 644 & 4.2 & 11690 & 76.3 & 1796 & 11.7 & 1172 & 7.7 \\
\hline 5 & 17719 & 15.2 & 687 & 3.9 & 13308 & 75.1 & 2157 & $12.2 \%$ & 1567 & 8.8 \\
\hline 6 & 19291 & 16.5 & 687 & 3.6 & 14885 & 77.1 & 2304 & $11.9 \%$ & 1415 & 7.3 \\
\hline 7 & 15769 & 13.5 & 503 & 3.2 & 12382 & 78.5 & 1805 & $11.4 \%$ & 1079 & 6.8 \\
\hline 8 & 9506 & 8.1 & 278 & 2.9 & 7569 & 79.6 & 1107 & 11.6 & 552 & 5.8 \\
\hline 9 & 5743 & 4.9 & 181 & 3.2 & 4610 & 80.3 & 664 & 11.6 & 288 & 5.0 \\
\hline 10 & 1400 & 1.2 & 39 & 2.8 & 1125 & 80.4 & 164 & 11.7 & 72 & 5.1 \\
\hline Girls & 56678 & & 2082 & 3.7 & 44201 & 78.0 & 6674 & 11.8 & 3721 & 6.6 \\
\hline \multicolumn{11}{|c|}{ SEP } \\
\hline 1 & 2224 & 3.9 & 173 & 7.8 & 1769 & 79.5 & 178 & 8.0 & 104 & 4.7 \\
\hline 2 & 4698 & 8.3 & 277 & 5.9 & 3734 & 79.4 & 453 & 9.6 & 234 & 5.0 \\
\hline 3 & 8710 & 15.4 & 436 & 5.0 & 6781 & 77.8 & 963 & 11.1 & 530 & 6.1 \\
\hline 4 & 7308 & 12.9 & 265 & 3.6 & 5635 & 77.1 & 894 & 12.2 & 514 & 7.0 \\
\hline 5 & 8666 & 15.3 & 280 & 3.2 & 6537 & 75.4 & 1099 & 12.7 & 750 & 8.7 \\
\hline 6 & 9421 & 16.6 & 273 & 2.9 & 7307 & 77.5 & 1183 & 12.6 & 658 & 7.0 \\
\hline 7 & 7661 & 13.5 & 195 & 2.5 & 6015 & 78.5 & 924 & 12.1 & 527 & 6.9 \\
\hline 8 & 4556 & 8.0 & 107 & 2.3 & 3644 & 79.9 & 562 & 12.3 & 243 & 5.3 \\
\hline 9 & 2747 & 4.8 & 65 & 2.4 & 2214 & 80.6 & 341 & 12.4 & 127 & 4.6 \\
\hline 10 & 687 & 1.2 & 11 & 1.6 & 565 & 82.2 & 77 & 11.2 & 34 & 4.9 \\
\hline Boys & 60096 & & 2904 & 4.8 & 46250 & 76.9 & 6532 & 10.9 & 4410 & 7.3 \\
\hline \multicolumn{11}{|l|}{ SEP } \\
\hline 1 & 2241 & 3.7 & 180 & 8.0 & 1771 & 79.0 & 178 & 7.9 & 112 & 5.0 \\
\hline 2 & 5105 & 8.5 & 312 & 6.1 & 3994 & 78.2 & 487 & 9.5 & 312 & 6.1 \\
\hline 3 & 9066 & 15.1 & 589 & 6.5 & 6833 & 75.3 & 950 & 10.5 & 694 & 7.7 \\
\hline 4 & 7994 & 13.3 & 379 & 4.7 & 6055 & 75.7 & 902 & 11.3 & 658 & 8.2 \\
\hline 5 & 9053 & 15.1 & 407 & 4.5 & 6771 & 74.8 & 1058 & 11.7 & 817 & 9.0 \\
\hline 6 & 9870 & 16.4 & 414 & 4.2 & 7578 & 76.8 & 1121 & 11.4 & 757 & 7.7 \\
\hline 7 & 8108 & 13.5 & 308 & 3.8 & 6367 & 78.5 & 881 & 10.9 & 552 & 6.8 \\
\hline 8 & 4950 & 8.2 & 171 & 3.5 & 3925 & 79.2 & 545 & 11.0 & 309 & 6.2 \\
\hline 9 & 2996 & 5.0 & 116 & 3.9 & 2396 & 80.0 & 323 & 10.8 & 161 & 5.4 \\
\hline 10 & 713 & 1.2 & 28 & 3.9 & 560 & 78.5 & 87 & 12.2 & 38 & 5.3 \\
\hline
\end{tabular}

SEP, socioeconomic position.

uniformly distributed; $3.8 \%$ of children were in the lowest SEP (SEP 1), 1.2\% of children were in the highest SEP (SEP 10) and $74 \%$ of children were in SEPs 3-7 (table 1).

In 2018, 4.3\% of children were documented as being thin (table 1). Thinness was more common in boys compared with girls $(4.8 \%$ vs $3.7 \%)(p<0.001)$. Thinness rates decreased monotonically as SEP increased (table 1 and figure 1A). Prevalence of normal weight decreased between SEP 1 and SEP 5 and then showed an increasing trend (table 1 and figure 1B). Overweight prevalence was slightly higher in girls compared with boys $(11.8 \%$ vs $10.9 \%)$ ( $\mathrm{p}<0.001)$. Overweight rates increased between SEP 1 and SEP 5 and then remained relatively stable (table 1 and figure 1C). Obesity prevalence was slightly lower in girls compared with boys $(6.6 \%$ vs $7.3 \%)(\mathrm{p}<0.001)$. Obesity rates were highest in SEP 5 (girls 8.7\%, boys 9.0\%) and lowest in SEPs 1 and 10 (table 1 and figure 1D).

Focusing on the most recent year available (2018), we found considerable disparities in the rate of thinness (Lowest vs Highest SEP: Girls: OR 5.02, 99\% CI 2.23 to 11.30; Boys: OR 2.03,
99\% CI 1.19 to 3.48) (table 2). The association between SEP and overweight did not differ substantially between girls and boys $(p=0.78)$. Boys and girls in SEP 1 had lower odds of being overweight compared with those in SEP 10, although this association was only statistically significant in boys (Girls: OR $0.74,99 \% \mathrm{CI}$ 0.51 to 1.07 , Boys: OR $0.65,99 \%$ CI 0.45 to 0.93 ). The association between SEP and obesity was not linear, and the addition of a quadratic term significantly improved the goodness-of-fit of the model assessing the association between SEP and obesity, indicating an inverse U-shaped association. The odds of obesity in SEP 5 were nearly two times the odds of obesity in the highest SEP (Girls: OR ${ }_{\text {SEP } 5 \text { vs } 10} 1.91,99 \%$ CI 1.20 to 3.04 ; Boys: OR ${ }_{\text {SEP } 5}$ vs $101.78,99 \%$ CI 1.14 to 2.77$)$.

The prevalence of thinness did not change over time $(p=0.53)$ (online supplementary figure 1 ). We detected an increasing trend in normal weight $(74.5 \%$ in 2014 vs $77.4 \%$ in $2018, \mathrm{p}<0.001)$, while the prevalence of overweight and obesity decreased over time (Overweight: $13.3 \%$ to $11.3 \% ; \mathrm{p}<0.001$, Obesity: $8.0 \%$ to $7.0 \% ; \mathrm{p}<0.001)$. 

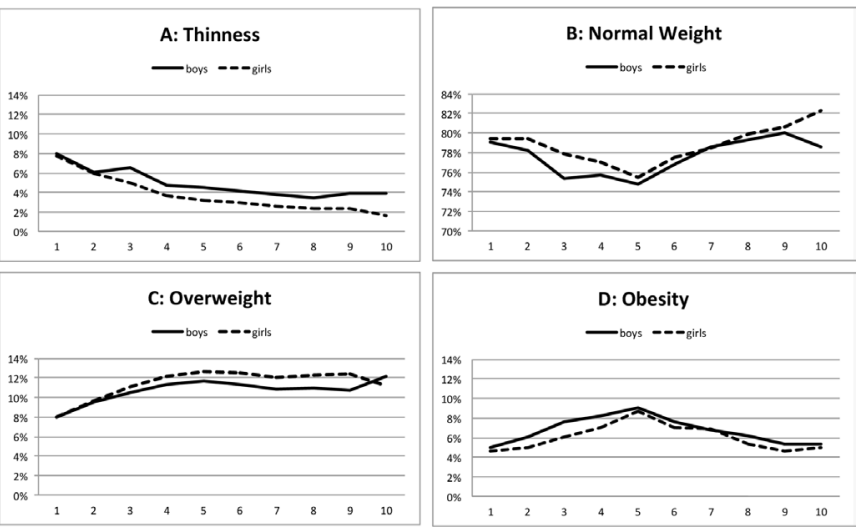

Figure 1 Nutritional status of 5-6-year-old boys and girls, by SEP, 2018, Israel ( $n=116774)$. (A) Thinness among boys and girls. (B) Normal weight among boys and girls. (C) Overweight among boys and girls. (D) Obesity among boys and girls. SEP, socioeconomic position.

SEP disparities in thinness increased for the first few years, followed by a decrease $(p=0.004)$ (table 2$)$. Although the change in disparities was not monotonic, among boys, the disparities were reduced from 3.4 in 2014 to 2.0 in 2018, while a reverse trend was seen in girls (2.7 in 2014, 5.0 in 2018). We found a weak trend in disparities for overweight, in boys and girls, and the disparities were not significant for most of the years we assessed. SEP disparities in obesity fluctuated over time in both girls and boys. Among girls, the disparities increased from 1.5 in 2014 to 1.9 in 2018, while in boys, the disparities in 2014 were very similar to those in 2018 (1.8 and 1.7, respectively).

\section{DISCUSSION}

Overweight and obesity prevalence rates decreased over time, while the prevalence of normal weight increased, with no change in thinness prevalence. Major SEP disparities were observed for thinness as well as for obesity. Over the 2014-2018 period, SEP disparities in thinness decreased for boys, but increased for girls. Disparities in overweight did not change over time. SEP disparities in obesity worsened slightly for girls and did not change for boys.

We found major disparities in thinness prevalence between the lowest SEP and all other SEPs. SEP 1 in our study is comprised entirely of the minority Bedouin population, which differs from other groups in Israeli society in terms of cultural norms, SEP and health-related behaviours and outcomes. The Bedouins living in Israel are a previously nomadic people, who have undergone a transition over the past 50 years. Notably, under the National Health Insurance law, the Bedouin population is covered by mandatory health insurance and has access to healthcare. ${ }^{21}$ Nearly $40 \%$ of Bedouins live in unrecognised villages, in which living conditions are especially poor. ${ }^{2}$ Bedouin children have higher rates of anaemia and longer hospitalisation periods for infectious diseases, ${ }^{22}$ but have higher rates of timely childhood vaccination ${ }^{23}$ and higher rates of height and weight documentation compared with the Jewish population. More generally, health utilisation patterns in Israel differ by SEP, with those in lower SEPs being more likely to see a primary care provider. ${ }^{24}$ A previous study among Bedouin children at 18 months of age found that although the rate of stunting decreased substantially between 1990 and 2010, it remained higher than the rate in the Jewish population. ${ }^{25}$ Bedouin children in Israel may experience a nutrition transition leading to the double burden of undernutrition and obesity at the same time, as has been found in children in various middle-income and lowincome countries and ethnic minorities. ${ }^{26} 27$

Most studies have found an inverse association between individual-level SEP and obesity in high-income countries; ${ }^{728}$ however, this association varies by gender, age and ethnicity. ${ }^{29} 30$ Both positive and negative associations have been reported for the association between area-level SEP and obesity, while others have found no association. ${ }^{10-1231}$ One US study found that while individual-level SEP was consistently inversely associated with

\begin{tabular}{|c|c|c|c|c|c|c|}
\hline & $2014(n=96405)$ & $2015(n=101575)$ & $2016(n=115157)$ & $2017(n=117937)$ & $2018(n=116774)$ & $P_{\text {Trend }}$ \\
\hline \multicolumn{7}{|l|}{ Total } \\
\hline Thinness $O R_{\text {SEP } 1 \text { vs SEP } 10}$ & $3.06(1.97$ to 4.76$)$ & 3.27 (2.05 to 5.20$)$ & 3.62 (2.38 to 5.49$)$ & $3.43(2.27$ to 5.21$)$ & 2.89 (1.85 to 4.50$)$ & 0.004 \\
\hline Normal weight OR & Ref & Ref & Ref & Ref & Ref & \\
\hline Overweight $0 \mathrm{R}_{\text {SEP } 1 \text { vs SEP } 10}$ & 0.84 (0.63 to 1.12$)$ & 0.79 (0.60 to 1.04$)$ & 0.99 (0.78 to 1.25$)$ & $0.78(0.60$ to 1.01$)$ & 0.69 (0.53 to 0.89$)$ & $<0.001$ \\
\hline Obesity $O R_{\text {SEP } 5 \text { v } \text { SEP } 10}^{*}$ & $1.66(1.22$ to 2.27$)$ & 1.74 (1.27 to 2.38$)$ & 1.62 (1.21 to 2.16$)$ & 1.53 (1.13 to 2.07$)$ & 1.84 (1.34 to 2.54$)$ & $<0.001$ \\
\hline \multicolumn{7}{|l|}{ Girls } \\
\hline Thinness $O \mathrm{R}_{\text {SEP } 1 \text { v s SEP } 10}$ & 2.67 (1.38 to 5.14) & 1.94 (1.08 to 3.48 ) & 2.80 (1.58 to 4.95$)$ & 4.34 (2.24 to 8.40$)$ & $5.02(2.23$ to 11.30$)$ & 0.02 \\
\hline Normal Weight OR & Ref & Ref & Ref & Ref & Ref & \\
\hline Overweight $O R_{\text {SEP } 1 \text { vs SEP } 10}$ & 0.63 (0.42 to 0.94$)$ & $0.80(0.54$ to 1.19$)$ & 0.74 (0.53 to 1.05$)$ & 0.77 (0.52 to 1.16$)$ & 0.74 (0.51 to 1.07 ) & 0.01 \\
\hline Obesity $0 R_{\text {SEP } 5 \text { vs SEP } 10}$ & 1.50 (0.97 to 2.34$)$ & 2.00 (1.21 to 3.31$)$ & 1.51 (0.98 to 2.33$)$ & 1.54 (0.98 to 2.42 ) & 1.91 (1.20 to 3.04$)$ & $<0.001$ \\
\hline \multicolumn{7}{|l|}{ Boys } \\
\hline Thinness $O \mathrm{R}_{\text {SEP } 1 \text { vs SEP } 10}$ & 3.44 (1.90 to 6.23$)$ & $6.10(2.78$ to 13.41$)$ & 4.62 (2.51 to 8.52 ) & 2.86 (1.67 to 4.90$)$ & 2.03 (1.19 to 3.48$)$ & $<0.001$ \\
\hline Normal weight OR & Ref & Ref & Ref & Ref & Ref & \\
\hline Overweight $0 R_{\text {SEP } 1 \text { vs SEP } 10}$ & $1.11(0.74$ to 1.67$)$ & $0.80(0.55$ to 1.16$)$ & 1.27 (0.91 to 1.77$)$ & $0.78(0.55$ to 1.10$)$ & 0.65 (0.45 to 0.93 ) & 0.01 \\
\hline Obesity $O R_{\text {SEP } 5 \text { v s SEP } 10}$ & 1.82 (1.19 to 2.80$)$ & 1.58 (1.05 to 2.36$)$ & 1.71 (1.16 to 2.52$)$ & 1.51 (1.00 to 2.27$)$ & 1.78 (1.14 to 2.77$)$ & 0.01 \\
\hline
\end{tabular}

Multinomial regression models used to assess the association between SEP and nutritional status, adjusted for sex. Multinomial model compared odds of thinness, overweight and obesity to normal weight.

$P_{\text {trend }}$ based on multinomial model with an interaction between SEP and time.

Values in bold indicate statistical significance $(p<0.01)$

*In obesity, instead of a monotonic trend, we found a reverse U-shaped association. To assess SEP disparities in this category, we compared the lower-middle SEP to the highest (5 vs 10), as SEP 5 consistently had the highest prevalence of obesity.

SEP, socioeconomic position. 
obesity, substantial geographic differences existed when using an area-level SEP measure. ${ }^{32}$ Several recent studies from Australia found large SEP disparities in obesity between children in low SEP versus children in high SEP. ${ }^{30} 33$ Higher prevalence of obesity was recently reported among Australian Indigenous children. Similarly, in the UK, the rates of obesity are highest among children in the lowest SEP and not in middle-lower SEPs. ${ }^{34}$ Some geographic areas displayed an inverse quadratic association, ${ }^{32}$ with highest obesity rates in the lower-middle SEPs, similar to our findings.

Our findings support the stabilisation in obesity prevalence rates found in European countries ${ }^{7}$ and even show a small decrease, similar to that found in the Iberian region. ${ }^{35}$ This reduction may be due to ongoing efforts of the Israeli ministries of health, education and culture and sports to combat obesity and sedentary lifestyles, with a national programme for active and healthy living. ${ }^{36}$ Our study did not find a decreasing trend in thinness, despite a reduction in the global age-standardised prevalence of thinness in both girls and boys. ${ }^{9}$

We did not find a reduction in SEP disparities in overweight and obesity prevalence and even found some increases. Changes in SEP disparities, particularly for thinness, may be influenced by the small number of children in the extreme SEP categories. Increases in SEP disparities have also been found in England, ${ }^{34}$ while no change in disparities were found in the US or France. ${ }^{3738}$

Our study included all children aged 5-6 in Israel and therefore our study was not prone to selection biases or sampling errors. To our knowledge, this is one of the first population studies to include detailed area-level SEP categories. The quality of our data was exceptionally high. Data were checked at three levels: an internal audit by each HMO, a QICH programme directorate data audit and an external process audit. ${ }^{15}$

However, weight and height measurements, which require human assessment and documentation, vary between testers and may be subject to dishonest reporting. In addition, we only had data from the last 5 years and therefore we were unable to assess trends over a longer period.

Children in lower SEPs may not be reaping the greatest benefit from national obesity-prevention efforts and healthy nutrition programmes operated by local HMOs and NGOs operating in Israel. Efforts are necessary to further develop multisectoral sustainable partnerships to enable healthy nutrition for children of low and middle-low SEP.

Correction notice This article has been updated since it was published online. An acknowledgement has been added.

Twitter Yiska Loewenberg Weisband @yiskaw

Acknowledgements The authors wish to thank the Israel National Institute for Health Policy Research for their partial funding of this research.

Contributors YLW and OM conceived and designed the study. VKS, YWS, MK and WAA collected the data. YLW, VKS, YWS, MK, WAA and OM contributed data or analysis tools. YLW performed the analysis. YLW wrote the paper, with considerate input from VKS, YWS, MK, WAA and OM.

Funding This work was supported by the Azrieli Foundation, by awarding Yiska Loewenberg Weisband an Azrieli Postdoctoral fellowship.

Competing interests YLW reports grants from Azrieli Foundation, during the conduct of the study.

Patient consent for publication Not required.

Provenance and peer review Not commissioned; externally peer reviewed.

Data availability statement Data are available on reasonable request. Data is available with the corresponding author.

ORCID iD

Yiska Loewenberg Weisband http://orcid.org/0000-0002-5064-5902

\section{REFERENCES}

1 Sanders RH, Han A, Baker JS, et al. Childhood obesity and its physical and psychological co-morbidities: a systematic review of Australian children and adolescents. Eur J Pediatr 2015;174:715-46.

2 Treister-Goltzman Y, Peleg R. What is known about health and morbidity in the pediatric population of Muslim Bedouins in southern Israel: a descriptive review of the literature from the past two decades. I Immigr Minor Health 2015;17:940-6.

3 Laitinen J, Kiukaanniemi K, Heikkinen J, et al. Body size from birth to adulthood and bone mineral content and density at 31 years of age: results from the Northern Finland 1966 birth cohort study. Osteoporos Int 2005;16:1417-24.

4 Zhao Y, Wang Z, Yang J, et al. [Burden of disease caused by low body weight in children aged. Chinese J Epidemiol 2015;36:1279-82.

5 Serdula MK, Ivery D, Coates RJ, et al. Do obese children become obese adults? A review of the literature. Prev Med 1993;22:167-77.

$6 \mathrm{Li} \mathrm{J}$, Eriksson M, He W, et al. Associations between childhood body size and seventeen adverse outcomes: analysis of 65,057 European women. Sci Rep 2017;7:16917.

7 Chung A, Backholer K, Wong E, et al. Trends in child and adolescent obesity prevalence in economically advanced countries according to socioeconomic position: a systematic review. Obes Rev 2016;17:276-95.

8 O'Dea JA, Dibley MJ. Prevalence of obesity, overweight and thinness in Australian children and adolescents by socioeconomic status and ethnic/cultural group in 2006 and 2012. Int J Public Health 2014;59:819-28.

9 NCD Risk Factor Collaboration (NCD-RisC). Worldwide trends in body-mass index, underweight, overweight, and obesity from 1975 to 2016: a pooled analysis of 2416 population-based measurement studies in 128.9 million children, adolescents, and adults. Lancet 2017;390:2627-42.

$10 \mathrm{Kim} \mathrm{Y,} \mathrm{Cubbin} \mathrm{C,} \mathrm{Oh} \mathrm{S.} \mathrm{A} \mathrm{systematic} \mathrm{review} \mathrm{of} \mathrm{neighbourhood} \mathrm{economic} \mathrm{context} \mathrm{on}$ child obesity and obesity-related behaviours. Obes Rev 2019;20:420-31.

11 Lee H, Harris KM, Lee J. Multiple levels of social disadvantage and links to obesity in adolescence and young adulthood. J Sch Health 2013;83:139-49.

12 Pardo-Crespo MR, Narla NP, Williams AR, et al. Comparison of individuallevel versus area-level socioeconomic measures in assessing health outcomes of children in Olmsted County, Minnesota. J Epidemiol Community Health 2013:67:305-10.

13 Cole TJ, Flegal KM, Nicholls D, et al. Body mass index cut offs to define thinness in children and adolescents: international survey. BMJ 2007;335:194.

14 World Health Organization (WHO). Growth reference 5-19 years. World Health Organization, 2007

15 Manor 0, Shmueli A, Ben-Yehuda A, et al. Israel national program for quality indicators in community healthcare. English summary report. 2011-2015. Available: http://healthindicators.org.il/wp-content/uploads/2014/05/English-report-20112015_final.pdf

16 Israeli Ministry of Health. A guide to assessing growth and nutritional status from birth to age 6 years. Available: http://www.health.gov.il/Subjects/KidsAndMatures/ Pages/curves.aspx [Accessed 16 Jan 2020].

17 Wang Y, Chen H-J. Handbook of Anthropometry: Physical Measures of Human Form in Health and Disease. In: Preedy VR, ed. Handbook of anthropometry: physical measures of human form in health and disease. London: Springer Science \& Business Media, 2012: 29-48.

18 Central Bureau of Statistics Israel. Characterization and classification of geographical units by the socio-economic level of the population 2008. Jerusalem, 2013.

19 Points location intelligence, 2019. Available: http://www. points.co.l//?lang=en [Accessed 11 Jul 2019].

20 Manor O, Matthews S, Power C. Comparing measures of health inequality. Soc Sci Med 1997;45:761-71.

21 Rights under Law. Rights under Natl. heal. Insur. law.

22 Levy A, Fraser D, Vardi H, et al. Hospitalizations for infectious diseases in Jewish and Bedouin children in southern Israel. Eur J Epidemiol 1998;14:179-86.

23 Stein-Zamir C, Israeli A. Age-appropriate versus up-to-date coverage of routine childhood vaccinations among young children in Israel. Hum Vaccin Immunother 2017:13:2102-10.

24 Filc D, Davidovich N, Novack L, et al. Is socioeconomic status associated with utilization of health care services in a single-payer universal health care system? Int J Equity Health 2014;13:115.

25 Rubin L, Belmaker I, Somekh E, et al. Maternal and child health in Israel: building lives. Lancet 2017;389:2514-30.

26 Tzioumis E, Adair LS. Childhood dual burden of under- and overnutrition in low- and middle-income countries: a critical review. Food Nutr Bull 2014;35:230-43.

27 Kain J, Uauy R, Lera L, et al. Trends in height and BMI of 6-year-old children during the nutrition transition in Chile. Obes Res 2005;13:2178-86.

28 Barriuso L, Miqueleiz E, Albaladejo R, et al. Socioeconomic position and childhoodadolescent weight status in rich countries: a systematic review, 1990-2013. BMC Pediatr 2015;15:129.

29 Wang Y, Lim H. The global childhood obesity epidemic and the association between socio-economic status and childhood obesity. Int Rev Psychiatry 2012;24:176-88.

30 Hardy LL, Cosgrove C, King L, et al. Shifting curves? trends in thinness and obesity among Australian youth, 1985 to 2010. Pediatr Obes 2012;7:92-100. 


\section{Original research}

31 Navalpotro L, Regidor E, Ortega P, et al. Area-Based socioeconomic environment, obesity risk behaviours, area facilities and childhood overweight and obesity: socioeconomic environment and childhood overweight. Prev Med 2012;55:102-7.

$32 \mathrm{Jin} \mathrm{H}$, Lu Y. The relationship between obesity and socioeconomic status among Texas school children and its spatial variation. Appl Geogr 2017;79:143-52.

33 Hardy LL, Mihrshahi S, Gale J, et al. 30-Year trends in overweight, obesity and waistto-height ratio by socioeconomic status in Australian children, 1985 to 2015. Int J Obes 2017:41:76-82.

34 Stamatakis E, Wardle J, Cole TJ. Childhood obesity and overweight prevalence trends in England: evidence for growing socioeconomic disparities. Int I Obes 2010;34:41-7.
35 Garrido-Miguel M, Cavero-Redondo I, Álvarez-Bueno C, et al. Prevalence and trends of overweight and obesity in European children from 1999 to 2016: a systematic review and meta-analysis. JAMA Pediatr 2019:e192430.

36 Nutrition Department,. Ministry of health. Available: https://www.health.gov.il/ English/MinistryUnits/HealthDivision/PublicHealth/nutrition/Pages/default.aspx [Accessed 25 Oct 2018]

37 Rossen LM, Schoendorf KC. Measuring health disparities: trends in racial-ethnic and socioeconomic disparities in obesity among 2- to 18-year old youth in the United States, 2001-2010. Ann Epidemiol 2012;22:698-704.

38 Thibault H, Carriere C, Langevin C, et al. Evolution of overweight prevalence among 5-6-year-old children according to socio-economic status. Acta Paediatr 2013:102:273-7. 